\title{
From Mesohepatectomy to Mini-Mesohepatectomy: Evolving the Concept of Resectability of Hepatic Tumors at the Hepatocaval Confluence
}

\author{
Matteo Donadon Guido Torzilli \\ Liver Surgery Unit, Third Department of General Surgery, University of Milan, IRCCS Istituto Clinico Humanitas, \\ Rozzano, Italy
}

\section{Key Words}

Mini-mesohepatectomy $\cdot$ Mesohepatectomy $\cdot$ Hepatic resection - Intraoperative ultrasound - Middle hepatic vein $\cdot$ Hepatocaval confluence $\cdot$ Hepatic tumors

\begin{abstract}
In the case of tumors in contact with or invading the middle hepatic vein (MHV) at the hepatocaval confluence, extended right hepatectomy or mesohepatectomy is usually recommended. Major or extended hepatectomy is associated with significant rates of morbidity and mortality, and a more conservative approach would be desirable. Thus, we developed a new conservative operation, the so-called mini-mesohepatectomy that, in some specific circumstances, allowed the en-bloc resection of segment 8 , segment 4-sup and the $\mathrm{MHV}$ at the hepatocaval confluence and at the same time preservation of the remaining parenchyma of the right anterior section and left median section drained by the MHV. The present work describes the rationale, indications, as well as the surgical technique of this new operation which we believe should be part of the armamentarium of the modern hepatic surgeon, and will probably limit the need for a formal mesohepatectomy.

Copyright $\odot 2011$ S. Karger AG, Basel
\end{abstract}

\section{Introduction}

Mesohepatectomy is a major central hepatectomy involving the removal of segments 5,8 , and 4 , and resection of the middle hepatic vein (MHV) at the hepatocaval confluence. First described by Hasegawa et al. [1], this complex operation usually requires a full dissection of the hepatic hilum to isolate and ligate the arterial supports and the glissonian branches to the right anterior section (segments 5-8) as well as to the left median section (segment 4). Traditionally, when tumors invade the MHV at the confluence into the inferior vena cava (IVC), a right trisectionectomy is performed by most surgeons, and mesohepatectomy is reserved by others as a conservative alternative to the previously cited operation [2-5]. However, as major or extended hepatectomies - even in experts hands - are associated with significant rates of morbidity and mortality [6-9], a more conservative approach even in such complex tumor presentation is desirable.

Based on a solid knowledge of intraoperative ultrasound (IOUS) and ultrasound-guided hepatic resection aimed to preserve functional liver parenchyma $[10,11]$, we developed a new ultrasound-guided conservative resection, the so-called mini-mesohepatectomy $(\mathrm{MMH})$ [12].

\section{KARGER \\ Fax +4161306 1234 \\ E-Mail karger@karger.ch}

www.karger.com (c) $2011 \mathrm{~S}$. Karger AG, Basel

$0253-4886 / 11 / 0282-0109 \$ 38.00 / 0$

Accessible online at:

www.karger.com/dsu
Guido Torzilli, MD, PhD

Liver Surgery Unit, Third Department of Surgery

University of Milan, IRCCS Istituto Clinico Humanitas

Via Manzoni 56, IT-20089 Rozzano (Italy)

Tel. +39028224 4083,E-Mail guido.torzilli@unimi.it 
Fig. 1. a EF-IOUS scan shows blood flow into the MHV (arrow). b EF-IOUS scan, taken at the time the MHV has been clamped by the surgeon's finger compression $(\mathrm{F})$, shows that there is no more blood flow (no color signal) into the MHV.
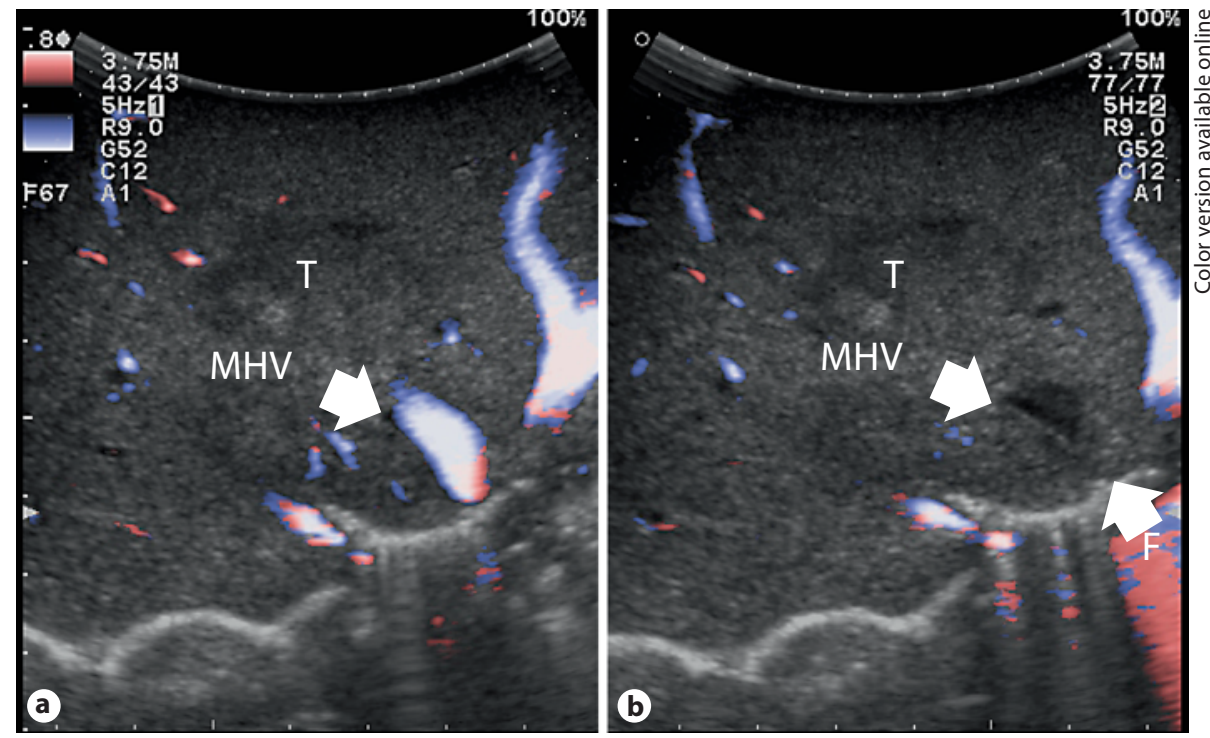

\section{Methods}

\section{Definitions}

The terminology for liver anatomy and resections used in this work is based on the Brisbane classification [13]. MMH is defined as the partial removal of segments 8 and 4 superior including the involved tract of MHV.

\section{Indications}

Candidates for the MMH are patients with tumors with indefinite margins or direct evidence of invasion of MHV at the hepatocaval confluence, which are defined as the tract of the vein within $4 \mathrm{~cm}$ from the IVC. Of course, patients with bulky tumors occupying the right anterior section (segments 5-8) and segment 4 , as well as patients with invasion of another hepatic vein (right or left hepatic vein), cannot receive this approach.

\section{Operative Technique}

Patients are prepared in a standard manner, and positioned in the supine position on the operating table. J-shaped laparotomy incision is generally carried out with the aim to control the hepatocaval confluence by fingertip compression, as described below. Access to the thoracic cavity following the 9th intercostal space is carried out when the control at the hepatocaval confluence is felt as inadequate especially in obese patients, in patients with deep chest, and in reoperation. After a partial mobilization of the liver, IOUS is performed using an Aloka Alpha 10 (Aloka Itd; Tokyo, Japan) equipped with the standard 2- to 6-MHz convex probe, 5- to 10-MHz T-shaped probe, and with the 5- to 10 $\mathrm{MHz}$ microconvex probe. The intraoperative staging is completed by contrast-enhanced IOUS, using the dedicated 1.88 - to $3.76-\mathrm{MHz}$ harmonic frequency probe, in those patients with doubtful and/or new findings based on our previously reported criteria $[14,15]$.

At this point, the anterior surface of the hepatocaval confluence is exposed, and the space between the right hepatic vein (RHV) and MHV at the confluence into the IVC is obtained to

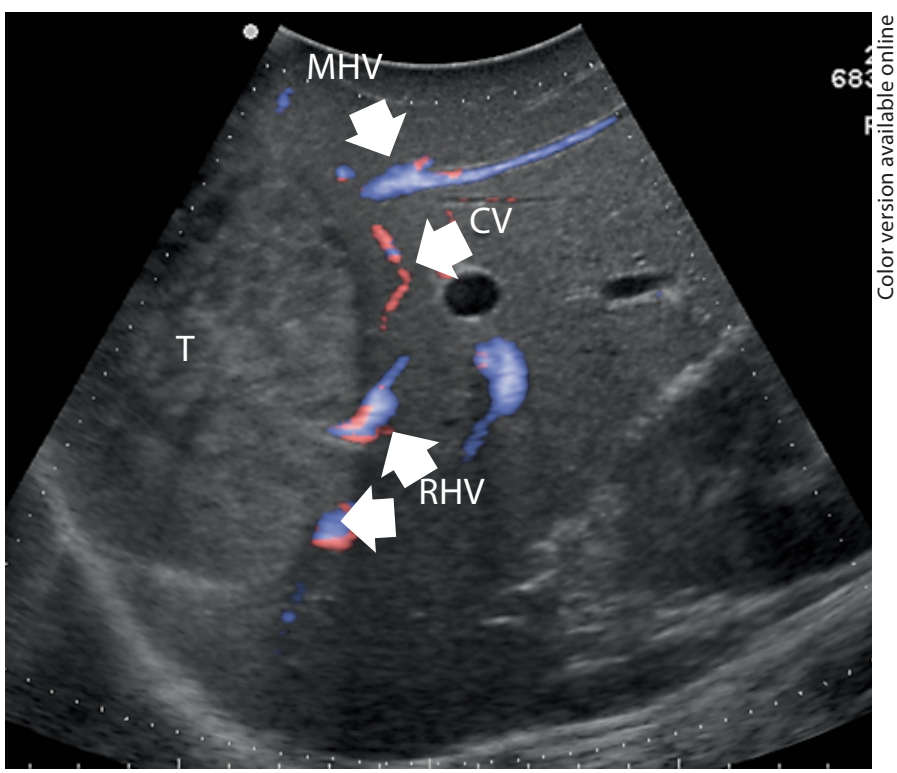

Fig. 2. EF-IOUS scan shows flow in a communicating vein $(\mathrm{CV})$ connecting the MHV and the RHV. T = Tumor.

accommodate the surgeon's fingertip. Fingertip compression is then applied to the MHV to test the feasibility of MMH.

\section{IOUS Criteria for $M M H$}

To perform MMH, at least one of these three criteria should be confirmed once the fingertip is compressing the MHV (fig. 1):

(1) Reversal flow direction in the peripheral portion of the MHV, which suggests drainage through collateral circulation in adjacent hepatic veins or IVC.

(2) Detectable shunting collaterals between MHV and right or left hepatic vein (fig. 2). 


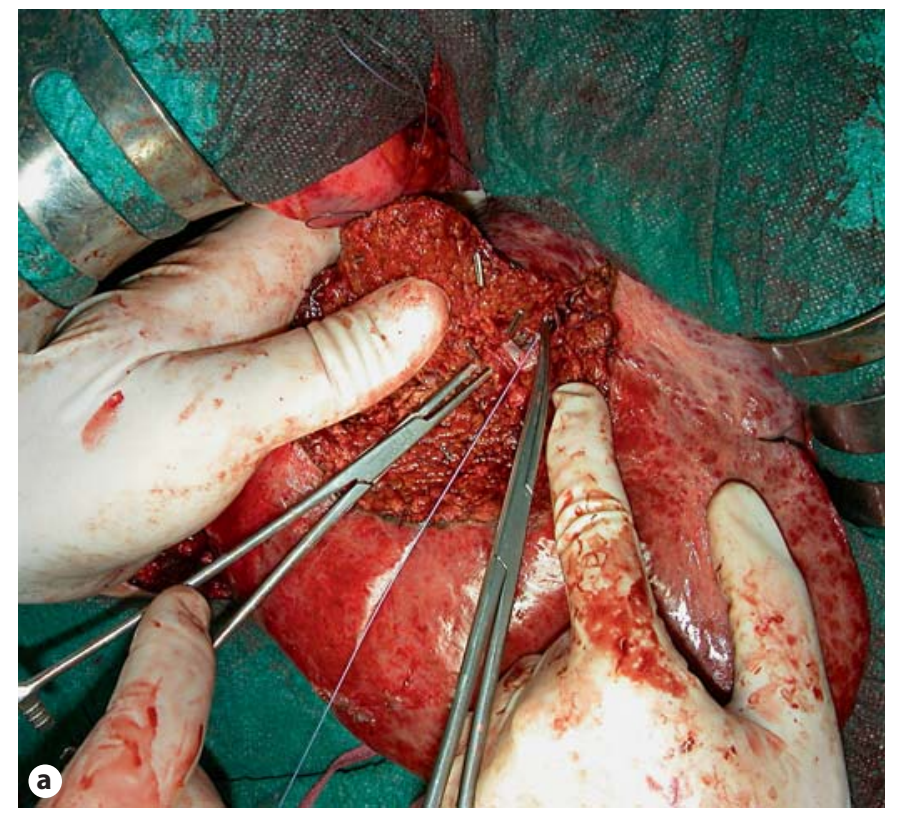

Fig. 3. a Position of surgeon's hands during liver dissection: the surgeon's left hand sustains the right hemiliver, with the 1st finger keeping the dissection area opened, the 2 nd finger positioned on the posterior aspect of the line drawn with the electrocautery on the glissonian capsule and the 3rd finger positioned in the fossa

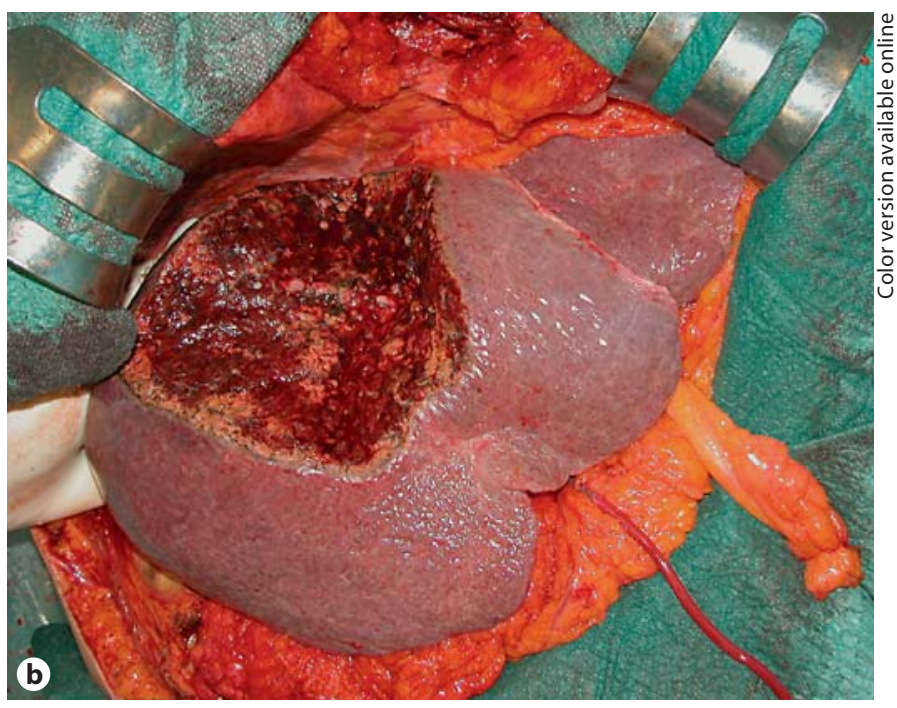

between RHV and MHV caval insertion for backflow bleeding control in the event it would be needed. $\mathbf{b}$ The resection area at the end of the $\mathrm{MMH}$ results in a parenchyma-sparing hepatectomy, but with a flat and smooth cut surface.

under IOUS control, the electrocautery between the probe and the liver surface allows to define the caudal and lateral portions of the resection area, while the surgeon's fingertip pushing the liver from the most cranial portion is visualized on the ultrasound scan and allows to define the cranial limit of the resection area, and then the dissection plane. Electrocautery is then used to draw the resection area on the liver surface. Dissection is carried out with the surgeon's left hand sustaining the right hemiliver with his 1st finger keeping the dissection area opened, the 2nd finger positioned on the posterior aspect of the line drawn with the electrocautery on the glissonian capsule and the 3rd finger positioned in the fossa between RHV and MHV caval insertion for backflow bleeding control in the event it would be needed (fig. 3a). The main objective, once the resection area is drawn, is to achieve the flattest and smoothest cut surface (fig. 3b). The posterior wall of the MHV or of the tumor itself is used as the deepest landmark of the resection area. IOUS guidance is used to follow the dissection plane getting closer to the targeted point for MHV division.

\section{Discussion}

The rate of major hepatic resections represents approximately half of the resections performed in most centers, and the associated mortality and morbidity are not negligible [6-9]. Therefore, the need to reduce that rate 
should be one of the priorities in hepatic surgery, and indeed some authors have proposed alternative approaches in selected patients [19-22]. However, in the case of tumor touching or infiltrating the MHV, most of the authors would suggest performing extended right hepatectomy, probably with preoperative portal vein embolization, while others might consider mesohepatectomy, which is a valid conservative alternative.

Following our previously reported studies, in which we have shown the feasibility and reliability of 'the radical but conservative approach' for liver tumors with complex vascular relations $[10,11]$, we developed $\mathrm{MMH}$, a new operation that further enhances the concept of parenchyma sparing in liver surgery [12].

It could be argued that the preservation of part of the right anterior section and of the left median section without the MHV represents a risk for venous congestion that might be a source of morbidity. In fact, based on previous studies, only $24 \%$ of patients had communicating veins between MHV and RHV, and just $43 \%$ of patients who received the right liver without MHV after living donor liver transplantation had no residual congested areas based on color Doppler evaluation of portal branch blood flow direction [22]. However, we did not experience a single case of congestion, and this is strictly related to the extensive use of EF-IOUS which has allowed the disclosure of communicating veins between adjacent hepatic veins in most patients with tumor located at the hepatocaval confluence. Indeed, in a recent report we have shown how communicating veins appear on EF-IOUS in $80 \%$ of such patients [23].

A second concern that might be raised regards the surgical margin. From the oncological standpoint, many studies have previously reported that the width of the surgical margin was not correlated with the true recurrence rate $[24,25]$. In particular, the zero margin in the case of hepatocellular carcinoma did not increase the risk of recurrence as shown by our previous reports $[10,11]$, and as recently confirmed by Matsui et al. [25] in a large series of patients. On the other hand, also in the case of liver metastases, the $0-\mathrm{mm}$ margin is progressively becoming acceptable $[26,27]$. When oncologically acceptable, the parenchyma sparing policy has allowed us to perform surgery in a single session in cases of multiple bilobar colorectal cancer liver metastases [28]. These patients need to be radically operated on with a single procedure. If they are carriers of lesions located at the hepatocaval confluence, they will benefit from the combination of procedures such as the herein described $\mathrm{MMH}$ and the previously reported SERPS. This is an example of an ap- plication of $\mathrm{MMH}$ which makes otherwise unfeasible surgical approaches feasible, at least in a single procedure. Certainly, a parenchyma-sparing technique such as $\mathrm{MMH}$, when oncologically acceptable, would be a welcomed option in cases where the suitability of the surgical treatment of hepatocellular carcinoma has to be evaluated. Indeed, although a hepatocellular carcinoma invading a hepatic vein is a less frequent occurrence when compared with metastastic lesions, there is room for the surgical treatment anyway [29], and for that the suitability of a parenchyma-sparing option such as MMH could be crucial for the decision to proceed or not.

In conclusion, $\mathrm{MMH}$ for removal of tumor in segment 8 or segment 4 -sup that is in contact with or infiltrating the MHV is a feasible, safe and effective operation, and we believe should be part of the armamentarium of the modern hepatic surgeon. Its application will probably drastically limit the use of formal mesohepatectomy. The latter is somehow controversial due to the technically demanding double-cut surface, and the insufficient benefit in terms of parenchyma sparing. Formal mesohepatectomy could be now be replaced by a technique which promotes a drastic limitation of parenchyma removal (fig. 3). Application of $\mathrm{MMH}$ is more versatile; it can be used also in combination with other resection and offer a better solution for the surgical treatment of patients with complex presentations.

\section{Disclosure Statement}

The authors have nothing to disclose.

References

1 Hasegawa H, Makuuchi M, Yamazaki S, Gunvén P: Central bisegmentectomy of the liver: experience in 16 patients. World J Surg 1989;13:786-790.

2 Wu CC, Ho WL, Chen JT, Tang CS, Yeh DC, Liu TJ, et al: Mesohepatectomy for centrally located hepatocellular carcinoma: an appraisal of a rare procedure. J Am Coll Surg 1999;188:508-515.

-3 Scudamore CH, Buczkowski AK, Shayan $\mathrm{H}$, Ho SG, Legiehn GM, Chung SW, Owen DA: Mesohepatectomy. Am J Surg 2000;179:356360.

$4 \mathrm{Hu}$ RH, Lee PH, Chang YC, Ho MC, Yu SC: Treatment of centrally located hepatocellular carcinoma with central hepatectomy. Surgery 2003;133:251-256.

-5 Stratopoulos C, Soonawalla Z, Brockmann J, Hoffmann K, Friend PJ: Central hepatectomy: the golden mean for treating central liver tumors? Surg Oncol 2007;16:99-106. 
6 Kooby DA, Stockman J, Ben-Porat L, Gonen M, Jarnagin WR, Dematteo RP, et al: Influence of transfusions on perioperative and long-term outcome in patients following hepatic resection for colorectal metastases. Ann Surg 2003;237:860-869.

$>7$ Jarnagin WR, Gonen M, Fong Y, DeMatteo $\mathrm{RP}$, Ben-Porat L, Little S, et al: Improvement in perioperative outcome after hepatic resection: analysis of 1,803 consecutive cases over the past decade. Ann Surg 2002;236:397406.

$>8$ Bismuth H, Chiche L, Adam R, Castaing D, Diamond T, Dennison A: Liver resection versus transplantation for hepatocellular carcinoma in cirrhotic patients. Ann Surg 1993;218:145-151.

9 Grazi GL, Ercolani G, Pierangeli F, Del Gaudio M, Cescon M, Cavallari A, et al: Improved results of liver resection for hepatocellular carcinoma on cirrhosis give the procedure added value. Ann Surg 2001;234: 71-78.

-10 Torzilli G, Montorsi M, Donadon M, Palmisano A, Del Fabbro D, Gambetti A, et al: 'Radical but conservative' is the main goal for ultrasonography-guided liver resection: prospective validation of this approach. J Am Coll Surg 2005;201:517-528.

- 11 Torzilli G, Montorsi M, Del Fabbro D, Palmisano A, Donadon M, Makuuchi M: Ultrasonographically guided surgical approach to liver tumours involving the hepatic veins close to the caval confluence. Br J Surg 2006; 93:1238-1246.

12 Torzilli G, Palmisano A, Procopio F, Cimino M, Botea F, Donadon M, et al: A new systematic small for size resection for liver tumors invading the middle hepatic vein at its caval confluence: mini-mesohepatectomy. Ann Surg 2010;251:33-39.

13 Terminology Committee of the IHPBA: Terminology of liver anatomy and resections. HPB Surg 2000;2:333-339.
14 Torzilli G, Palmisano A, Del Fabbro D, Marconi M, Donadon M, Spinelli A, et al: Contrast-enhanced intraoperative ultrasonography during surgery for hepatocellular carcinoma in liver cirrhosis: is it useful or useless? A prospective cohort study of our experience. Ann Surg Oncol 2007;14:1347-1355.

15 Torzilli G, Botea F, Procopio F, Donadon M, Balzarini L, Lutman F, et al: Does contrastenhanced intraoperative ultrasonography impact radicality of hepatectomies for colorectal cancer liver metastases in spite of modern preoperative imaging? Analysis on a prospective cohort. Eur J Cancer 2008;6:1623.

16 Makuuchi M, Hasegawa H, Yamazaki S: Intraoperative ultrasonic examination for hepatectomy. Jpn J Clin Oncol 1981;11:367-390.

17 Torzilli G, Makuuchi M: Ultrasound-guided liver subsegmentectomy: the peculiarity of segment 4. J Am Coll Surg 2001;193:706708 .

18 Torzilli G Procopio F, Cimino M, Del Fabbro D, Palmisano A, Donadon M, et al: Anatomical segmental and subsegmental resection of the liver for hepatocellular carcinoma: a new approach by means of ultrasound-guided vessel compression. Ann Surg 2010;251: 229-235.

19 Chouillard E, Cherqui D, Tayar C, Brunetti F, Fagniez PL: Anatomical bi- and trisegmentectomies as alternatives to extensive liver resections. Ann Surg 2003;238:29-34.

20 Torzilli G, Donadon M, Marconi M, Botea F, Palmisano A, Del Fabbro D, et al: Systematic extended right posterior sectionectomy: a safe and effective alternative to right hepatectomy. Ann Surg 2008;247:603-611.

-21 Sano K, Makuuchi M, Miki K, Maema A, Sugawara Y, Imamura H, et al: Evaluation of hepatic venous congestion: proposed indication criteria for hepatic vein reconstruction. Ann Surg 2002;236:241-247.

22 Poon RT, Fan ST, Ng IO, Wong J: Significance of resection margin in hepatectomy for hepatocellular carcinoma: a critical reappraisal. Ann Surg 2000;231:544-551.
23 Torzilli G, Garancini M, Donadon M, et al: Intraoperative ultrasonographic detection of communicating veins between adjacent hepatic veins during hepatectomy for tumours at the hepatocaval confluence. $\mathrm{Br}$ J Surg 2010;97:1867-1873.

24 Ochiai T, Takayama T, Inoue K, Yamamoto J, Shimada K, Kosuge T, et al: Hepatic resection with and without surgical margins for hepatocellular carcinoma in patients with impaired liver function. Hepatogastroenterology 1999;46:1885-1889.

25 Matsui Y, Terakawa N, Satoi S, et al: Postoperative outcomes in patients with hepatocellular carcinomas resected with exposure of the tumor surface: clinical role of the nomargin resection. Arch Surg 2007;142:596602.

26 Pawlik TM, Scoggins CR, Zorzi D, Abdalla EK, Andres A, Eng C, Curley SA, Loyer EM, Muratore A, Mentha G, Capussotti L, Vauthey JN: Effect of surgical margin status on survival and site of recurrence after hepatic resection for colorectal metastases. Ann Surg 2005;241:715-722.

27 de Haas RJ, Wicherts DA, Flores E, Azoulay $\mathrm{D}$, Castaing D, Adam R: R1 resection by necessity for colorectal liver metastases: is it still a contraindication to surgery? Ann Surg 2008;248:626-637.

28 Torzilli G, Procopio F, Botea F, et al: Onestage ultrasonographically guided hepatectomy for multiple bilobar colorectal metastases: a feasible and effective alternative to the 2-stage approach. Surgery 2009;146:60-71.

29 Torzilli G, Donadon N, Marconi M, Palmisano A, Del Fabbro D, Spinelli A, Botea F, Montorsi M: Hepatectomy for hepatocellular carcinoma in stage B and C of Barcelona Clinic Liver Cancer Classification: results of a prospective analysis. Arch Surg 2008;143: 1082-1090. 